\title{
Establishment and CT Imaging of Rabbits Abdominal Aorta Atherosclerosis Model Based on High-fat Diet and Balloon Strain Technique
}

\author{
Dawei Wang ${ }^{1, \dagger}$, Tao Yang ${ }^{2}{ }^{\dagger}$, Xiangyi Chen ${ }^{3}$, Feng Guo ${ }^{1}$, Liujun $\mathrm{Jia}^{2}$, Guangxin Yue ${ }^{2}$, \\ Ying Kui Liang, ${ }^{1, *}$ Xin Wang ${ }^{2, *}$ \\ ${ }^{1}$ Department of Nuclear Medicine, The Sixth Medical Center of PLA General Hospital, Beijing, People's Republic of China \\ ${ }^{2}$ Department of Cardiac Surgery, Department of Radiology, Fu Wai Hospital, National Center of Cardiovascular Diseases, Chinese Academy of \\ Medical Sciences \& Peking Union Medical College, Beijing, People's Republic of China \\ ${ }^{3}$ Department of Nuclear Medicine, the First College of Clinical Medical Sciences, China Three Gorges University, Yichang Central People's \\ Hospital, Yichang, People's Republic of China
}

\author{
Email address: \\ fuwaiwangxin@188.com (Xin Wang), liangyingkui2012@sina.com (Ying Kui Liang) \\ ${ }^{*}$ Corresponding author \\ $\dagger$ Dawei Wang and Tao Yang are co-first authors.
}

\section{To cite this article:}

Dawei Wang, Tao Yang, Xiangyi Chen, Feng Guo, Liujun Jia, Guangxin Yue, Ying Kui Liang, Xin Wang. Establishment and CT Imaging of Rabbits Abdominal Aorta Atherosclerosis Model Based on High-fat Diet and Balloon Strain Technique. International Journal of Biomedical Science and Engineering. Vol. 9, No. 3, 2021, pp. 73-77. doi: 10.11648/j.ijbse.20210903.14

Received: July 29, 2021; Accepted: September 11, 2021; Published: September 23, 2021

\begin{abstract}
Background and Objectives: Atherosclerosis is the most common type of arteriosclerotic vascular disease. It is characterized by accumulation of lipids, hemorrhage and thrombosis, and gradual degeneration and calcification of the middle layer of the artery. It is very harmful to human body. To diagnose atherosclerosis at an early stage, a new animal model of abdominal aorta in New Zealand rabbits was established using high-fat diet with balloon injury to simulate the natural process of human disease. Methods: In our study, the high-fat diet and balloon strain technique were used to establish this model, CT imaging and pathological examination were used to prove the successful establishment of the model. Results: The results demonstrated that two weeks after high-fat feeding, the rabbits' survival rate was $100 \%$ and their body weights gradually increased over time. Compared with basic levels, all atherosclerotic indexes (AI) were higher than 4. Pathological observation and CT imaging showed that the location of vascular injuries was stenosis and the lesions were consistent with the basic characteristics of atherosclerosis. Conclusions: The above results indicated that under our experimental conditions, the rabbits' model of abdominal aorta atherosclerosis (AS) could be successfully reproduced. Compared with previous atherosclerosis models, it has the characteristics of a short modeling time and method simplicity. More importantly, it can be used as a follow-up model of atherosclerosis early diagnosis.
\end{abstract}

Keywords: Atherosclerosis, Rabbits, Balloon Endothelial Injury, Animal Model, CT Imaging

\section{Introduction}

In recent years, cardiovascular and cerebrovascular diseases increased and become the main cause of human death, even outranking cancers [1-3]. Studies have shown that atherosclerosis (AS) is the cause of the most important risk factors of cardiovascular and cerebrovascular diseases [4-6]. Therefore, it is of great significance to establish a clinical animal model for the study of human cardiovascular and cerebrovascular diseases [7]. Ignatowski [8] firstly reported, that feeding animals with protein-rich food, could successfully induce aortic intimal lesions in rabbits. Since then, it has gradually increased to establish models that mimic human diseases in small rodents (rats, mice and hamsters), birds 
(chickens, pigeons), pigs, carnivores (dogs, cats) and non-human primates [9-10]. Forster and haunstetter [11] made a comparative study of the basic pathology and pathophysiology of atherosclerotic plaques between rabbits and humans and found that the metabolism of blood lipid between rabbits and humans is quite similar. Meanwhile, in rabbits, the absorption rate of intestinal cholesterol is high, but the clearance ability of plasma cholesterol is low [11]. Hence, to establish an atherosclerotic model in rabbits is relatively simple and stimulate real forming process of atherosclerotic plaques in human bodies to a greater extent. However, when establishing atherosclerotic animal models, large doses of cholesterol are used for feeding. It may easily cause multiple organ lipid deposition, serious fatty liver, cholecystitis and pancreatitis and generate depilation, toenail fester, xanthoma and high blood viscosity, which are not conducive to the animal welfare [12]. In our study, we accelerated the formation of the atherosclerosis model by generating an advance endothelial damage and supplementing with high-fat diet. The purpose of this study was to establish a new abdominal aorta atherosclerosis model using high fat diet combined with balloon endothelial injury. CT imaging and pathological examination were used to evaluate the effectiveness of the model.

\section{Materials and Methods}

\subsection{Selection and Grouping of Experimental Animals}

A total of 20 New Zealand white rabbits with a weight of $3.0-4.4 \mathrm{~kg}$ were purchased from the same unit. The animals were randomly selected according to the feeding sequence of the animal house and numbered 1-20 in turn. They were randomly divided into 2 groups, Group A: normal control group; Group B: balloon treated group. The New Zealand white rabbits were fed with high fat diet $(21 \%$ fat and $1.5 \%$ $2.0 \%$ cholesterol feed) for 12 weeks. No restriction on water intake was applied in 4 groups.

\subsection{Generating a Vulnerable Plaque Model of Abdominal Aorta (Group B)}

After basic anesthesia (intramuscular injection of ketamine $1 \mathrm{ml} / \mathrm{kg}$ + diazepam $0.7 \mathrm{~mL} / \mathrm{kg}$ ), $3 \mathrm{~cm}$ incision was made in the right groin area and a $2 \mathrm{~cm}$ long femoral artery was separated. The femoral artery was punctured by Seldinger's method, the $5 \mathrm{~F}$ artery sheath was placed and the $5 \mathrm{~F}$ Berman double lumen balloon catheter was sent to the aortic arch along the artery sheath. According to the diameter of the aortic lumen, $0.5-1.0 \mathrm{ml}$ gas was injected to fill the balloon at the tip of the catheter, which was repeatedly pulled up and down, and at this time, the balloon expansion pressure is approximatively 0.7-1.0 MPa. The main artery arch reached the bifurcation of the iliac artery at the distal end of the abdominal aorta. After feeling mild to moderate resistance, at the end of the balloon traction, the balloon is pulled out $15 \mathrm{~cm}$, with the speed of 5 $\mathrm{cm} / \mathrm{s}$ and the whole pulling process is completed in 3-5 seconds. The pullback speed was approximatively $5 \mathrm{~cm} / \mathrm{s}$ and was repeated 3 times. After withdrawing the catheter and sheath, the femoral artery was ligated, and the skin incision was sutured. The modeling diagram of New Zealand white rabbit is shown in figure 1 below.
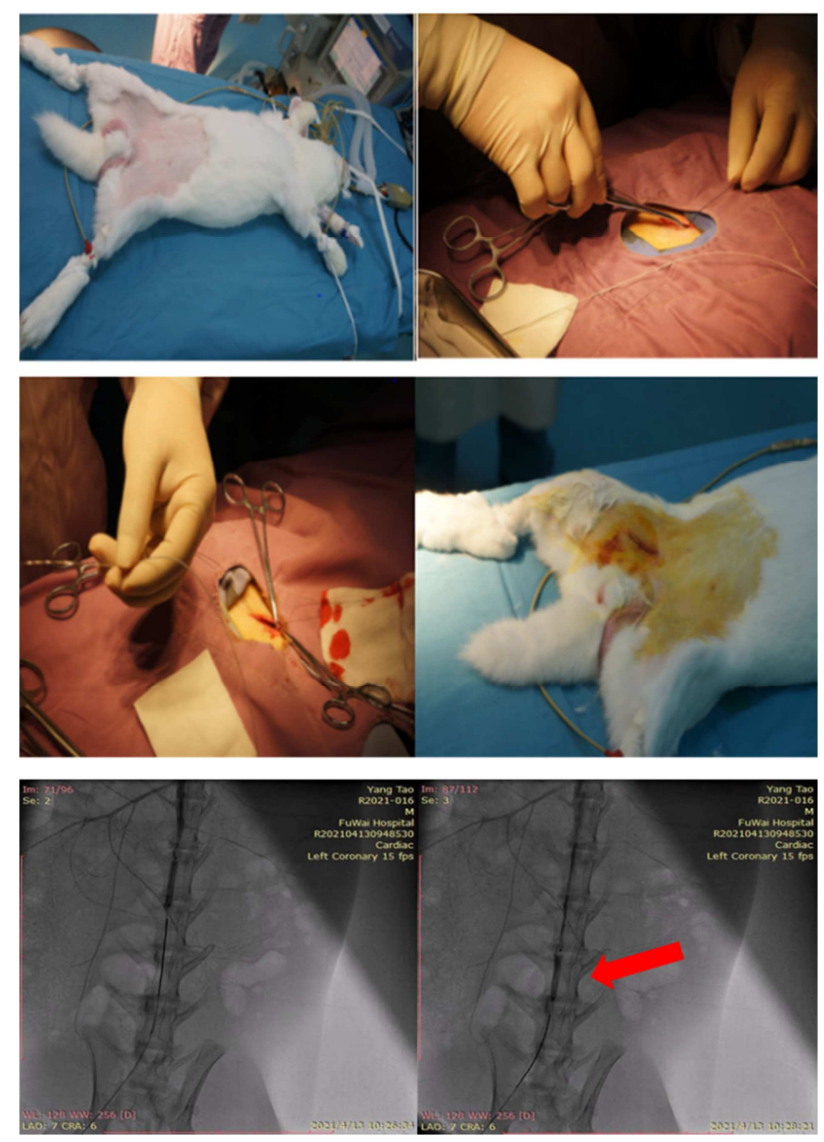

Figure 1. The modeling diagram of New Zealand white rabbit includes immobilization of animals, anesthesia and skin preparation; Free the femoral artery; The balloon was filled with gas (red arrow), and was pulled repeatedly in abdominal aorta for 3-5 times; The incision was sutured and the operation was finished.

\subsection{CT Equipment and Scanning Method}

All animals were sacrificed for pathological examination before using a 64 row MDCT enhanced scanning (Lightspeed VCT, GE) equipment to scan the abdominal aorta. The basic anesthesia was performed before CT examination in the experimental rabbits. After anesthesia, one ear vein was punctured, and $12 \mathrm{~g}$ trocar was placed. The non-ionic contrast agent iohexol $(350 \mathrm{mg} / 100 \mathrm{ml})$ was used for enhanced scanning. The volume ratio of iohexol and normal saline were used at 1:2, the dosage of the contrast agent was $8 \mathrm{ml}$, and the flow rate was $0.5 \mathrm{ml} / \mathrm{s}$. The head and tail of the rabbits were in supine position and the scanning range was from the aortic arch to the bifurcation of the iliac artery. The scanning parameters used were tube voltage $100 \mathrm{kV}$, tube current $100 \mathrm{Ma}$, collimator 64 layers $\times 0.625$ $\mathrm{mm}$, pitch 0.984:1, scanning layer thickness $0.625 \mathrm{~mm}$.

\subsection{MDCT Image Analysis}

All CT images were transmitted to the workstation (AW 4.3, GE) and the original images were reconstructed by the unified 
standard reconstruction algorithm. The image field of view was $160 \mathrm{~mm} \times 160 \mathrm{~mm}$ and the pixel matrix was $512 \times 512 \mathrm{~mm}$. The window width was set to $200 \mathrm{Hu}$ and the window level was set to $100 \mathrm{Hu}$. At first, the diagnosis was made without knowing the pathological results and then compared with the pathological results. A retrospective study was carried out to measure the plaque (aortic wall) thickness, CT value and other indicators. The normal aortic wall could not be displayed. Once the aortic wall was observed, it was regarded as thickening. The atherosclerotic lesions are shown as the bright contrast filling area in the middle (i.e. the lumen), surrounded by the fainter "halo" (i.e. the thickened aortic wall), or by the protruding plaque into the lumen, with an irregular aortic wall that lost its normal smooth shape.

\subsection{Pathological Examination}

All experimental rabbits were killed by intravenous injection of $10 \% \mathrm{KCl}$ on the second day after MDCT examination and was intravenously injected of the anticoagulant heparin (100U/kg) before the animals' death. The aortas were removed from their aortic arches to the lower parts of the bifurcation of the common iliac artery. Each aortic specimen was taken from the opening of the left subclavian artery, as the proximal anatomical mark, and from the foot side, at every interval of $1 \mathrm{~cm}$, including the opening of the right renal artery to the upper part of the bifurcation of the iliac artery, as the distal anatomical mark, with a distance of $1.0 \mathrm{~cm}$ from the head to the tail. The circular layer was not removed, and the details were recorded to correspond with the $\mathrm{CT}$ scanning layer. After paraffin embedding and fixation, the specimens were sectioned at $5 \mathrm{~m}$ thickness and stained with hematoxylin eosin (HE) and elastic fiber dye. After staining, it was judged by pathological experts whether there was a plaque formation and what characteristics the tissue was. According to the proportion of plaque lipid and fiber components, the plaque was defined as lipid-based or fiber-based plaque, and the intimal thickness was measured layer by layers.

\subsection{Statistical Analysis}

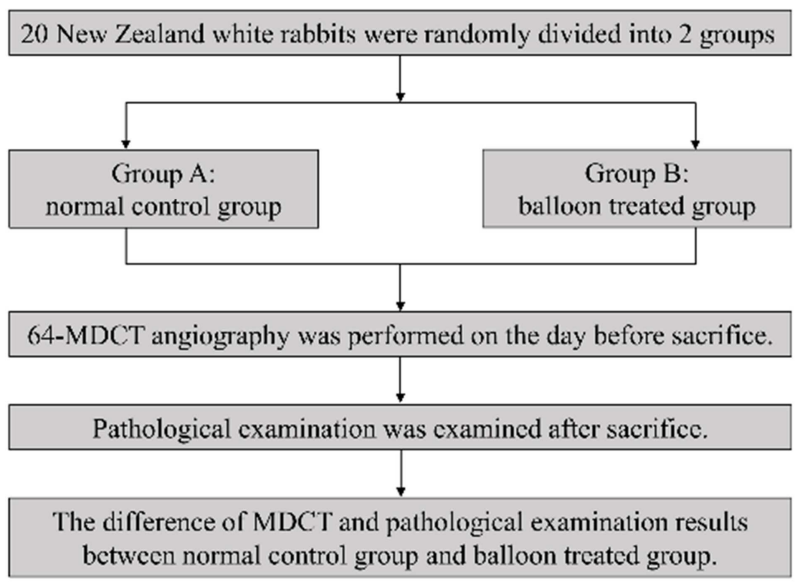

Figure 2. The white rabbits were randomly divided into 2 groups (normal control group and balloon treated group), 64-MDCT angiography and pathological examination were performed.
The SAS 9.1.3 software was used for statistical analysis. After consulting statistical experts, reasonable statistical methods were used to calculate the difference of pathological characteristics of plaque between normal group and balloon treated group. The correlation and difference between the MDCT results and the pathological, were statistically tested. P $<0.05$, which was statistically significant. The whole workflow is showed in Figure 2.

\section{Results}

In our study, the abdominal aorta atherosclerosis model was successfully developed in rabbits of the balloon treated group. Regarding CT imaging, the thickness of abdominal aorta wall of these rabbits had increased compared to the normal control group (Figure 3). No significant difference was found in plaque location, size and the stenosis rate. On the other hand, the pathological examination confirmed the existence of atherosclerotic plaques and intimal thickening (Figure 4). For the plaques location, there was no significant difference of the size and stenosis rate of the lumens between the two groups. Therefore, our results showed that the new abdominal aorta atherosclerosis model was successfully established.

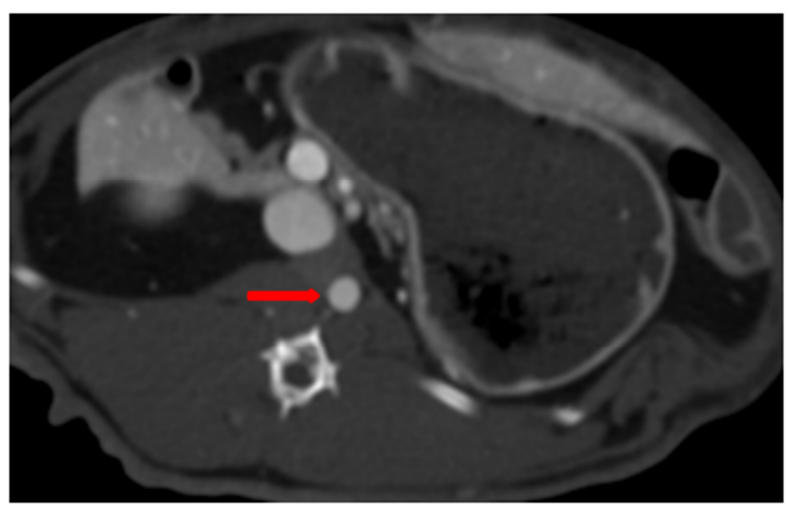

Figure 3. 64-MDCT showed that the thickness of abdominal aorta wall had increased in rabbits of balloon treated group.

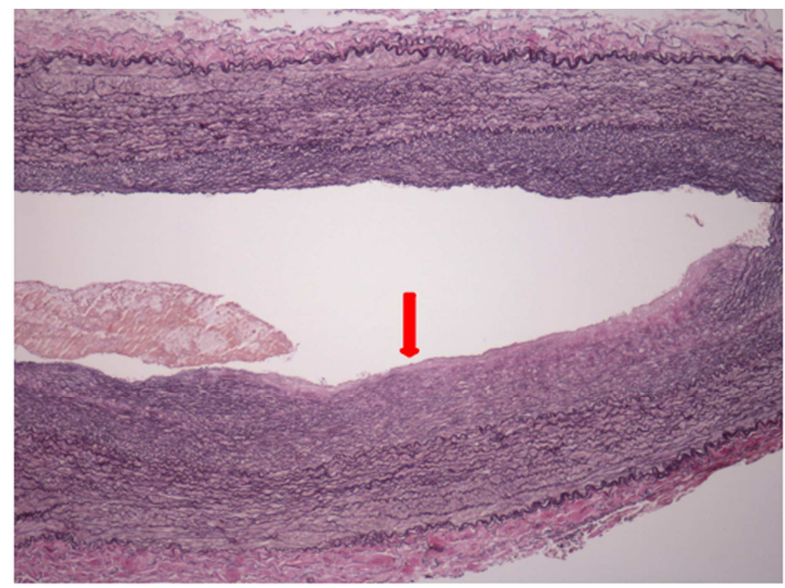

Figure 4. Pathological examination confirmed the formation of atherosclerotic plaques and the intimal thickening of the abdominal aorta. 


\section{Discussion}

In this study, New Zealand white rabbits were selected as the subjects, as they are sensitive to a high-fat diet. They are good models for the imitating the formation process of abdominal aorta atherosclerosis plaques formation in a hyperlipidemia environment, and for achieving the desired effect. To simplify the procedure of animal modeling and to increase the success rate, we created a modified method to develop this model. Finally, CT scan and pathological examination results confirmed the success of our atherosclerosis model.

The key points in the establishment of this animal model are the extent of balloon dilatation and the speed at which the balloon is repeatedly pulled back. If the balloon dilatated extensively or pilled back fast, the abdominal aorta is prone to rupture. However, if the balloon dilatation is slight, the intima cannot be fully damaged. From our experience, the balloon expansion pressure is approximatively $0.7-1.0 \mathrm{MPa}$ for best results. After feeling mild to moderate resistance, at the end of the balloon traction, the balloon is pulled out $15 \mathrm{~cm}$, with the speed of $5 \mathrm{~cm} / \mathrm{s}$ and the whole pulling process is completed in 3-5 seconds.

The pathological examination showed that the atherosclerosis plaques, including fibrous cap, calcification and fat core, are similar to those in humans. The pathological results also showed that the early plaques were mainly foam cells. After the lipid nucleus formation in the fibrous plaque, its central components are mainly cholesterol or cholesterol ester, containing solid crystalline and liquid crystalline. Ross et al [13] and Schwartz et al [14] indicated that they are three characteristics of atherosclerosis lesions: 1. proliferative smooth muscle cells, macrophages and lymphocytes; 2. connective tissue matrix formed by smooth muscle cells, including elastic fibrin, collagen and glycoprotein; 3 . accumulated lipids, free, or fixed cholesterol. In the present study, these typical pathological changes can definitely be seen in the abdominal aorta of the atherosclerosis animal model.

The established atherosclerotic model has the following advantages: New Zealand white rabbits are easy to raise and can mimic the formation of atherosclerosis in human vessels, such as the process of endothelium injury, thrombosis and lipid deposition. On the other hand, the establishing period is short, and it can be formed in 12 weeks after endothelial injury, much shorter than other atherosclerosis models. In summary, this atherosclerosis model has been established successfully, which is suitable for the study of anti-atherosclerotic drug therapy and early diagnosis of atherosclerosis plaques.

\section{Conclusion}

The method of high-fat diet combined with the balloon strain technique, to establish an abdominal aortic atherosclerosis model in rabbits, is simple and stable with a short-term cycle and high success rate. This experimental animal model could be used, not only for study of drug therapy of atherosclerosis, but also for early diagnosis and prevention of atherosclerosis lesions, which has a good application prospect. It is also suggested that follow-up or future work on this topic should be made.

\section{Limitations}

Certainly, our atherosclerosis model has certain limitations. First, because the atherosclerosis model has been successfully established for a short time, the model to drug sensitivity of anti-atherosclerotic drugs still needs to be studied in the future. Secondly, this model mainly aimed at mild atherosclerosis, as the lesion degree of the abdominal aorta atherosclerosis was limited. Thirdly, compared to angiography, CT imaging has advantages in terms of comfort, non-invasiveness and relative time of performance, while, there are still some potential problems, such as high operator dependence, poor reliability and difficulties with the localization of the target artery. Therefore, future studies need to address these problems to improve the atherosclerosis model.

\section{Compliance with Ethical Standards}

All protocols requiring the use of rabbits were approved by the Animal Care Committee of Fu Wai Hospital.

\section{Conflicts of Interest}

There are no conflicts of interest.

\section{Funding}

The study was supported by the Beijing Natural Science Foundation (grant no. 7204289), the Fundamental Research Funds for the Central Universities (grant no. 3332020020), the Innovation and cultivation fund of the sixth medical center of PLA General Hospital (grant no. CXPY202005), the Beijing Municipal Science and Technology Commission (grant nos. Z161100005016014 and Z101107052210004), the Beijing Key Laboratory of Pre-clinical Research and Evaluation for Cardiovascular Implant Materials (grant nos. 2018-PT2-ZR03 and 2018-PT2-ZR04), the Jilin Province Science \& Technology Committee (grant no. 20180101194JC), the Department of Education of Jilin Province (no. JJKH20200449KJ), the Science and Technology Innovation and Development Projects of Jilin City (grant no. 20190601178).

\section{Availability of Data and Materials}

All data generated or analysed during this study are included in this published article.

\section{References}

[1] World Health Organization, 2009. Cardiovascular Diseases [J]. Accessed June 17, 2010. 
[2] Burke AP, Farb A, Malcom GT, et al: Plaque rupture and sudden death related to exertion in men withcoronary artery disease. JAMA 1999; 281: 921-926.

[3] D. Mozaffarian, E. J. Benjamin, A. S. Go, D. K. Arnett, M. J. Blaha, M. Cushman, S. R. Das, S. de Ferranti, J. -P. Després, H. J. Fullerton, V. J. Howard, M. D. Huffman, C. R. Isasi, M. C. Jiménez, S. E. Judd, B. M. Kissela, J. H. Lichtman, L. D. Lisabeth, S. Liu, R. H. Mackey, D. J. Magid, D. K. McGuire, E. R. Mohler, C. S. Moy, P. Muntner, M. E. Mussolino, K. Nasir, R. W. Neumar, G. Nichol, L. Palaniappan, D. K. Pandey, M. J. Reeves, C. J. Rodriguez, W. Rosamond, P. D. Sorlie, J. Stein, A Towfighi, T. N. Turan, S. S. Virani, D. Woo, R. W. Yeh, M. B. Turner, Heart disease and stroke statistics-2016 update, Circulation (2015).

[4] Cui Wenyu, Du ran. Study on the animal model of carotid atherosclerosis Progress [J]. China urban and rural enterprise health, 2016 (6): 31-32.

[5] Leber AW, von Ziegler F, Becker A et al (2008) Characteristics of coronary plaques before angiographic progression determined by multi-slice CT. Int J Cardiovasc Imaging 24: 423-428.

[6] P. Libby, Inflammation in atherosclerosis, Nature 420 (6917) (2002) 868-874.

[7] A. G. Horti, Y. Gao, H. Kuwabara, Y. Wang, S. Abazyan, R. P. Yasuda, T. Tran, Y. Xiao, N. Ahibzada, D. P. Holt, K. J. Kellar, M. V. Pletnikov, M. G. Pomper, D. F. Wong and R. F. Dannals,
18F-ASEM, a radiolabeled antagonist for imaging the $\alpha 7$-nicotinic acetylcholine receptor with PET, JNucl. Med. 55 (4) (2014) 672-677.

[8] Chen Hua, Xie Zhongchen, Huang Guangyong, et al. Atherosclerosis in Wuzhishan miniature pig Establishment of metamodel. Experimental animal science, 2007, 24 (6): 39-43.

[9] Bergen W G, Mersanmann H J. Comparative aspect of lipid metabolism: import on contemporary research and use of animal models [J]. Journal of Nutrition. 2005, 135 (11): 2499-2502.

[10] Forster BA, Weinberg PD. Changes with age in the influence of endogenous nitricoxide on transport ProPerties of therabbit aortic wall near branches. Arterioscler Thromb Vasc Biol. 1997; 17: 1361-1368.

[11] Haunstetter A, Izumo S. Apoptosis: Basic mechanisms and implicatio ns for cardiovasculardisease. Circ Res. 1998; 82: 1111-1129.

[12] FAN J, SHIMOYAMADA H, SUN H, et al. Transgenic rabbits expressing human apolipoprotein (a) develop more extensive atherosclerotic lesions in response to a cholesterol-rich diet. Arterioscler Thromb Vascular Biol, 2001, 21: 88-94.

[13] Ross R. The pathogenesis of atherosclerosis: a perspective for the 1990s. Nature. 1993; 362 (6423): 801-809.

[14] Schwartz CJ, Valente AJ, Sprague EA. A modern view of atherosclerosis. Am J Card. 1993; 71 (6): B9-B14. 